\title{
Aceitabilidade alimentar do gafanhoto Cornops aquaticum (Bruner, 1906) (Orthoptera: Acrididae) na Amazônia oriental, Brasil Food acceptability of Cornops aquaticum (Bruner, 1906) (Orthoptera: Acrididae) in the Eastern Amazon, Brazil
}

\author{
Arthur Ferreira de Oliveiral', Ana Lúcia Nunes-Gutjahrl', Carlos Elias de Souza Bragal \\ 'Universidade do Estado do Pará. Belém, Pará, Brasil
}

\begin{abstract}
Resumo: Cornops aquaticum é um gafanhoto neotropical, semiaquático, de coloração predominantemente verde, que vive associado a macrófitas aquáticas da família Pontederiaceae, ocorrentes em corpos d'água amazônicos, especificamente as espécies do gênero Eichhornia. O Parque Estadual do Utinga (PEUt), Belém, Pará, vem sofrendo constantes infestações de macrófitas, principalmente de E. crassipes, nos seus lagos Bolonha e Água Preta, os quais abastecem de água o município de Belém. Este trabalho teve por objetivo realizar testes de aceitabilidade alimentar com o gafanhoto $C$. aquaticum. Para isso, foram ofertadas 12 espécies de macrófitas (mais abundantes no PEUt), como fonte alimentar para 240 adultos e 240 imaturos (ninfas), separadamente em béqueres de $250 \mathrm{ml}$, por um período de 21 dias, com uma repetição. Entre as 12 espécies testadas, C. aquaticum apresentou aceitação (aceitabilidade positiva) pelas espécies E. crassipes, Nothoscordum sp. e Pistia stratiotes. A maior sobrevivência de adultos e imaturos (ninfas) do gafanhoto, entre as três plantas aceitas, foi observada para E. crassipes. Durante o experimento, a maior mortalidade de C. aquaticum ocorreu nas macrófitas Hymenachne amplexicaulis, Nymphoides indica e Nymphaea ampla, indicando que estas plantas, entre as consumidas, parecem ser mais nocivas para o gafanhoto.
\end{abstract}

Palavras-chave: Gafanhoto semiaquático. Aceitação alimentar. Macrófitas aquáticas.

Abstract: Cornops aquaticum is a neotropical predominantly green grasshopper, semiaquatic, coloring, living associated with the macrophytes of Pontederiaceae family, found in Amazonian bodies of water, specifically the genus Eichhornia. The State Park Utinga (PEUt), Belém, Pará, has suffered constant infestations of weeds, especially E. crassipes in its Bolonha and Água Preta lakes, which supply water to the city of Belém. This work aimed to make food acceptability tests with the grasshopper C. aquaticum. For this, 12 macrophytes (more abundant in PEUt) as a food source for 240 and 240 immature adults (nymphs) separately in $250 \mathrm{ml}$ beakers were offered for a period of 21 days, with one repetition. Among the 12 species tested C. aquaticum showed acceptance (positive acceptability) for the species E. crassipes, Nothoscordum sp. and Pistia stratiotes. The higher survival of adult and immature (nymphs) of grasshopper accepted among the three plants was observed for $E$. crassipes. During the experiment the higher mortality of $C$. aquaticum occurred in macrophytes Hymenachne amplexicaulis, Nymphoides indica and Nymphaea ampla indicating that these plants, which were consumed, are the most harmful to the grasshopper.

Keywords: Semiaquatic grasshopper. Acceptance feeding. Aquatic macrophytes.

OLIVEIRA, A. F., A. L. NUNES-GUTJAHR \& C. E. S. BRAGA, 2016. Aceitabilidade alimentar do gafanhoto Cornops aquaticum (Bruner, 1906) (Orthoptera: Acrididae) na Amazônia oriental, Brasil. Boletim do Museu Paraense Emílio Goeldi. Ciências Naturais 10(2): 267-277. Autor para correspondência: Ana Lúcia Nunes Gutjahr. Universidade do Estado do Pará. Programa de Pós-Graduação em Ciências Ambientais. Trav. Enéas Pinheiro, 2626 - Marco. Belém, PA, Brasil. CEP 66095-100 (melcam@uol.com.br).

Recebido em 18/02/2014

Aprovado em 09/03/2016

Responsabilidade editorial: Mário Augusto G. Jardim

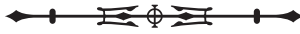




\section{INTRODUÇÃO}

Cornops aquaticum (Bruner, 1906) é um gafanhoto semiaquático, neotropical, que pode ser encontrado desde o sudeste do México até a Argentina (Adis et al., 2007; Capello et al., 2011). Essa espécie possui tamanho corpóreo variando de 2,5 a $3 \mathrm{~cm}$ de comprimento e padrão cromático verde-escuro, com faixa pós-ocular de coloração preta e amarela que se estende nas laterais do corpo (Lhano et al., 2005; Braga et al., 2011; Nunes-Gutjahr \& Braga, 2011). C. aquaticum é comumente encontrado em populações de macrófitas aquáticas pertencentes à família Pontederiaceae, dos gêneros Eichhornia Kunth e Pontederia L., nas quais desenvolve seu ciclo vital (Ferreira \& Vasconcellos-Neto, 2001; Franceschini et al., 2011).

É um gafanhoto que possui oviposição endofítica (Zolessi, 1956; Nunes-Gutjahr \& Braga, 2011) e apresenta uma série de adaptações para o ambiente aquático (Bentos-Pereira \& Lorier, 1991), pois vive sob plantas flutuantes, especificamente sob as espécies Eichhornia crassipes (Mart.) Solms e E. azurea (Sw.) Kuntl (Silva et al., 2010; Braga et al., 2013). Quanto a isso, Hill \& Cilliers (1999) e Braga et al. (2013), em estudos realizados na África do Sul e na Amazônia central, respectivamente, admitem que esse gafanhoto pode ser um possível controlador dessas espécies de macrófitas.

Os lagos Bolonha e Água Preta do Parque Estadual do Utinga (PEUt) encontram-se infestados por diferentes espécies de macrófitas aquáticas, cuja mais abundante é E. crassipes, no lago Bolonha. Tal infestação é possivelmente decorrente da degradação ambiental, que atualmente vem assolando as áreas e os mananciais do PEUt, onde são frequentes os despejos de matéria orgânica nos lagos, provenientes das constantes invasões por moradias de baixa renda em sua área e por conjuntos habitacionais em seus arredores (Brito, 2009).

Considerando que os lagos mencionados abastecem a cidade de Belém com água potável, é indiscutível a importância deles para a população, por isso, o controle dessas macrófitas é imprescindível. Atualmente, o método de controle empregado corresponde à retirada mecânica das plantas (controle mecânico), o qual é oneroso aos cofres públicos, sendo ineficiente até o momento.

Pelo exposto, este trabalho objetivou realizar um estudo sobre a aceitabilidade alimentar do gafanhoto C. aquaticum para a determinação de sua preferência alimentar, a partir da oferta de diferentes espécies de macrófitas aquáticas, oriundas dos lagos Bolonha e Água Preta do PEUt, a fim de gerar informações que subsidiem estudos capazes de indicar esse gafanhoto como um possível agente de controle biológico dessas plantas.

\section{MATERIAL E MÉTODOS}

\section{COLETA DE CORNOPS AQUATICUM}

Os gafanhotos $C$. aquaticum utilizados nos experimentos deste estudo foram coletados no Parque Estadual do Utinga (PEUt), localizado na região metropolitana de Belém, nas coordenadas $01^{\circ} 26^{\prime}$ 02” S, 48 26' 47" W. Esse parque foi instituído como unidade de proteção integral pelo decreto $n^{\circ} 1.330 / 2008$ e se encontra subordinado à Secretaria de Estado de Meio Ambiente (SEMA). O PEUt possui 1.340 hectares de área florestada, abrigando rica fauna e flora silvestre remanescente, além dos lagos Água Preta e Bolonha, que abastecem mais de 1 milhão de habitantes da cidade de Belém (Brito, 2009).

Durante as coletas, foram realizadas excursões periódicas, de abril a junho de 2013, para a captura de espécimes adultos e imaturos (ninfas) de $C$. aquaticum (Figura 1). Também foram coletadas 12 espécies de macrófitas (mais abundantes) que se encontravam nos lagos do parque, para serem utilizadas nos testes de aceitabilidade alimentar do gafanhoto em estudo. As plantas coletadas foram: Eichhornia crassipes (Mart.) Solms (Pontederiaceae, mureru), Salvinia auriculata (Aubl.) (Salvinaceae, carrapatinho d'água), Pistia stratiotes L. (Araceae, alface d'água), Paspalum sp. (Poaceae, capim), Nymphoides indica L. O. Kuntze (Menyanthaceae, estrela branca), Nymphaea ampla (Salisb.) DC. (Nymphaeaceae, 
aguapé-da-flor-branca), Hymenachne amplexicaulis (Rudge) Nees (Poaceae, rabo-de-raposa), Ludwigia helminthorrhiza Mart. (Onagraceae), Nothoscordum sp. (Alliacaea, alhobravo), Eleocharis acutangula Roxb. (Cyperaceae, junco-detrês-quinas), Polygonum acuminatum Kunth (Polygonacaeae, pimenta d'água) e Polygonum hydropiperoides Michx (Polygonacaeae, pimenta-do-brejo).

As excursões para coleta dos gafanhotos e das plantas foram realizadas no lago Água Preta, visto que o lago Bolonha encontrava-se em atividade constante de controle mecânico de macrófitas aquáticas. Nas excursões, foi utilizado um barco com motor de popa, o qual conduziu a equipe de coletores até as colônias de macrófitas que se encontravam no lago. As coletas foram sistemáticas, desenvolvidas durante as primeiras horas do dia, com duração média de 4 horas. Para a captura dos gafanhotos, foram utilizadas redes entomológicas (puçá), as quais foram passadas na porção aérea (fora d'água) das plantas. Após a captura, os gafanhotos foram retirados manualmente do puçá e introduzidos em sacos plásticos, acondicionados em caixas térmicas de poliestireno para serem transportados vivos ao laboratório.

As macrófitas foram coletadas manualmente de acordo com sua disponibilidade no ambiente aquático, sendo posteriormente introduzidas em sacos plásticos. Todo material coletado foi transportado para a Universidade do Estado do Pará (UEPA), onde foram realizados os testes de aceitabilidade alimentar em insetário telado, que mantinha as condições naturais de umidade e temperatura.

\section{TESTE DE ACEITABILIDADE ALIMENTAR}

O teste de aceitabilidade alimentar consistiu em submeter 20 adultos e 20 imaturos de $C$. aquaticum a cada uma das 12 espécies de macrófitas aquáticas testadas, utilizando um total de 480 gafanhotos. Este experimento buscou determinar as plantas que fazem parte da dieta alimentar de $C$. aquaticum. Nos testes, foram utilizados béqueres de $250 \mathrm{ml}$, forrados com chumaço de algodão umedecido em água, tampado com filme plástico, e no interior deste foi

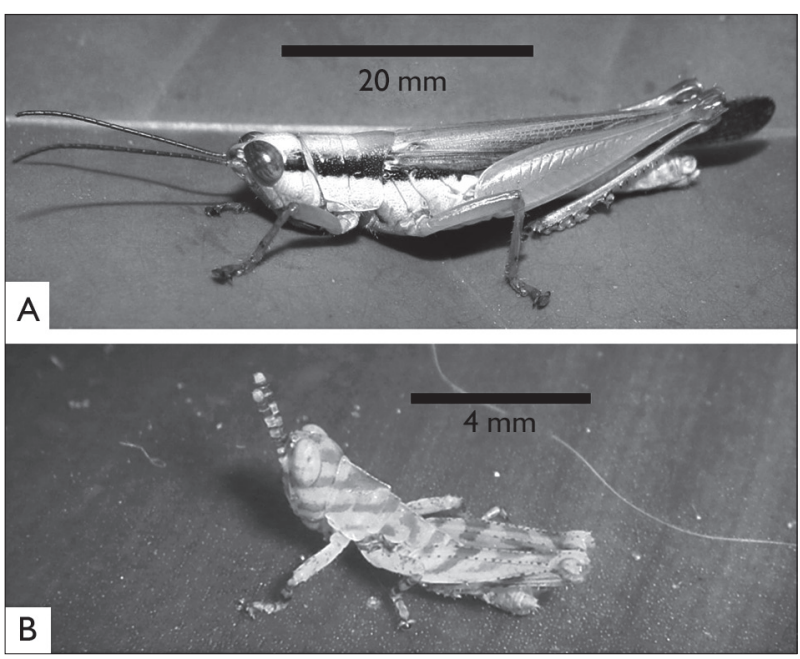

Figura 1. Gafanhoto Cornops aquaticum: A) adulto; B) imaturo (ninfa). Fotos: C. E. Braga, 2013.

introduzido um gafanhoto e uma folha (ou parte) da planta a ser testada (Nunes, 1989). Os béqueres foram dispostos em bandejas com água, para que o nível térmico no interior dos mesmos fosse mantido. $\bigcirc$ teste teve duração de 21 dias (Nunes, 1989), período compreendido para que ocorresse pelo menos um ou dois processos de muda dos imaturos, e teve observações diárias, quando era anotado se a planta foi consumida. A cada dois dias, foram feitas as trocas dos béqueres e das plantas correspondentes ao respectivo teste, quando eram retiradas as exúvias dos imaturos que mudaram e os gafanhotos mortos durante o experimento.

A aceitabilidade de C. aquaticum pelas plantas testadas levou em consideração a sobrevivência dos gafanhotos ao final do experimento, por isso, as plantas que apresentaram sobreviventes foram consideradas aceitas e tiveram aceitabilidade positiva (+), enquanto as que não apresentaram sobreviventes (não aceitas) tiveram aceitabilidade negativa (-).

Os gafanhotos mortos durante o experimento ficaram preservados em Eppendorf, com álcool 80\%, e foram depositados na Coleção Zoológica DidáticoCientífica da UEPA. Para todos os testes realizados, foi feita uma repetição para confirmação dos resultados.

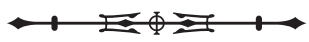




\section{RESULTADOS E DISCUSSÃO}

O gafanhoto $C$. aquaticum apresenta seletividade quanto à sua preferência por alimento, visto que, das 12 plantas testadas, houve a aceitação de três espécies de macrófitas - E. crassipes (Pontederiaceae), Nothoscordum sp. (Alliaceae) e Pistia stratiotes (Araceae) -, e a não aceitação de nove espécies. Tal resultado foi evidente para os adultos e imaturos testados (Tabelas 1 e 2). Ressalta-se que a aceitação de $E$. crassipes por $C$. aquaticum já foi obtida em outros estudos realizados com esse gafanhoto semiaquático (Adis \& Junk, 2003; Vieira \& Santos, 2003; Lhano et al., 2005; Capello et al., 2011; Franceschini et al., 2011), entretanto deve-se considerar que essa macrófita aquática pertence à família Pontederiaceae, a qual reúne os gêneros Pontederia e Eichhornia, que são considerados hospedeiros naturais de C. aquaticum (Zolessi, 1956; Roberts \& Carbonell, 1979; Guido \& Perkins, 1975; Hill \& Oberholzer, 2000).

Quanto à aceitação de Pistia stratiotes, vale ressaltar que essa espécie também foi aceita como alimentação por C. aquaticum em outro estudo na região amazônica (Vieira \& Santos, 2003), todavia, estudos realizados na Argentina por Capello et al. (2012) indicaram que esta macrófita aquática não faz parte da dieta alimentar do gafanhoto naquela região. Já em relação à aceitação da

Tabela 1. Sobrevivência de C. aquaticum (adultos) por dia de experimentação e aceitação em teste de aceitabilidade alimentar com 12 espécies de macrófitas aquáticas. Legendas: $\mathrm{N}=$ número de adultos testados em experimento com uma repetição; $\mathrm{X}=$ média de sobrevivência por dia; $\mathrm{Dp}=$ desvio padrão.

\begin{tabular}{|c|c|c|c|c|c|c|c|c|c|c|c|c|c|c|c|c|c|c|c|c|c|c|c|c|}
\hline \multirow{3}{*}{$\begin{array}{l}\text { Macrófitas } \\
\text { aquáticas }\end{array}$} & \multirow{3}{*}{$N$} & \multicolumn{23}{|c|}{ Número de dias de experimento } \\
\hline & & \multicolumn{2}{|c|}{$2^{\circ}$} & \multicolumn{2}{|c|}{$4^{\circ}$} & \multicolumn{2}{|c|}{$6^{\circ}$} & \multicolumn{2}{|c|}{$8^{\circ}$} & \multicolumn{2}{|c|}{$10^{\circ}$} & \multicolumn{2}{|c|}{$12^{\circ}$} & \multicolumn{2}{|c|}{$14^{\circ}$} & \multicolumn{2}{|c|}{$16^{\circ}$} & \multicolumn{2}{|c|}{$18^{\circ}$} & \multicolumn{2}{|c|}{$20^{\circ}$} & \multicolumn{2}{|c|}{$21^{\circ}$} & \multirow{2}{*}{ 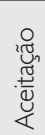 } \\
\hline & & $\times$ & คิ & $\times$ & $\stackrel{\circ}{0}$ & $\times$ & $\stackrel{\circ}{\circ}$ & $\times$ & อิ & $\times$ & ๑̊ & $\times$ & ๑̊ & $\times$ & $\stackrel{\circ}{0}$ & $\times$ & ๑̊ & $\times$ & ๑̊ & $\times$ & คิ & $\times$ & อิ & \\
\hline $\begin{array}{l}\text { Eichhornia } \\
\text { crassipes }\end{array}$ & 20 & 10 & 0 & 10 & 0 & 10 & 0 & 8.5 & 0.7 & 8.5 & 0.7 & 8.5 & 0.7 & 7.5 & 0.7 & 7 & 0 & 7 & 0 & 7 & 0 & 7 & 0 & + \\
\hline Paspalum sp. & 20 & 10 & 0 & 8.5 & 0.7 & 7 & 0 & 5 & 0 & 2 & 1.4 & 1 & 0 & 0 & 0 & 0 & 0 & 0 & 0 & 0 & 0 & 0 & 0 & - \\
\hline Pistia stratiotes & 20 & 10 & 0 & 9.5 & 0.7 & 6.5 & 0.7 & 5.5 & 0.7 & 5 & 0 & 5 & 0 & 4.5 & 0.7 & 3.5 & 0.7 & 3 & 1.4 & 3 & 1.4 & 3 & 1.4 & + \\
\hline $\begin{array}{l}\text { Polygonum } \\
\text { acuminatum }\end{array}$ & 20 & 10 & 0 & 5.5 & 0.7 & 1.5 & 0.7 & 0.5 & 0.7 & 0 & 0 & 0 & 0 & 0 & 0 & 0 & 0 & 0 & 0 & 0 & 0 & 0 & 0 & - \\
\hline $\begin{array}{l}\text { Hymenachne } \\
\text { amplexicaulis }\end{array}$ & 20 & 10 & 0 & 0 & 0 & 0 & 0 & 0 & 0 & 0 & 0 & 0 & 0 & 0 & 0 & 0 & 0 & 0 & 0 & 0 & 0 & 0 & 0 & - \\
\hline $\begin{array}{c}\text { Ludwigia } \\
\text { helminthorrhiza }\end{array}$ & 20 & 10 & 0 & 3.5 & 0.7 & 0.5 & 0.7 & 0 & 0 & 0 & 0 & 0 & 0 & 0 & 0 & 0 & 0 & 0 & 0 & 0 & 0 & 0 & 0 & - \\
\hline Nymphoides indica & 20 & 10 & 0 & 2.5 & 0.7 & 1 & 0 & 0 & 0 & 0 & 0 & 0 & 0 & 0 & 0 & 0 & 0 & 0 & 0 & 0 & 0 & 0 & 0 & - \\
\hline Nymphaea ampla & 20 & 10 & 0 & 1.5 & 0.7 & 0 & 0 & 0 & 0 & 0 & 0 & 0 & 0 & 0 & 0 & 0 & 0 & 0 & 0 & 0 & 0 & 0 & 0 & - \\
\hline Nothoscordum sp. & 20 & 10 & 0 & 8.5 & 0.7 & 8 & 0 & 8 & 0 & 7.5 & 0.7 & 6 & 1.4 & 5 & 1.4 & 5 & 0 & 5 & 0 & 5 & 0 & 5 & 0 & + \\
\hline $\begin{array}{l}\text { Eleocharis } \\
\text { acutangula }\end{array}$ & 20 & 10 & 0 & 6.5 & 0.7 & 3 & 1.4 & 1.5 & 0.7 & 0.5 & 0.7 & 0 & 0 & 0 & 0 & 0 & 0 & 0 & 0 & 0 & 0 & 0 & 0 & - \\
\hline Salvinia auriculata & 20 & 10 & 0 & 6.5 & 0.7 & 1.5 & 0.7 & 0.5 & 0.7 & 0 & 0 & 0 & 0 & 0 & 0 & 0 & 0 & 0 & 0 & 0 & 0 & 0 & 0 & - \\
\hline $\begin{array}{c}\text { Polygonum } \\
\text { hydropiperoides }\end{array}$ & 20 & 10 & 0 & 2 & 1.4 & 0.5 & 0.7 & 0 & 0 & 0 & 0 & 0 & 0 & 0 & 0 & 0 & 0 & 0 & 0 & 0 & 0 & 0 & 0 & - \\
\hline
\end{tabular}


espécie Nothoscordum sp. (Alliaceae), destaca-se que ela ainda não havia sido registrada como item alimentar de $C$. aquaticum, até o momento, caracterizando este estudo como o primeiro registro de aceitabilidade alimentar desse gafanhoto por esta espécie de macrófita.

A não aceitação das espécies Salvinia auriculata, Paspalum sp., Nymphoides indica, Nymphaea ampla, Hymenachne amplexicaulis, Ludwigia helminthorrhiza, Eleocharis acutangula, Polygonum acuminatum e Polygonum hydropiperoides pelo gafanhoto C. aquaticum como alimento não implica que os mesmos não tenham comido as plantas, pois em algumas delas a sobrevivência desses insetos (adultos e ninfas) estendeu-se até a $6^{\text {a }}$ observação (12 dias) de experimentação (Figura 2).

Esse resultado parece indicar que o gafanhoto em estado de ausência de alimento específico (com fome) tende a comer outras plantas, buscando saciar a falta de comida ou consumindo-as como fonte alimentar alternativa temporária.

Tabela 2. Sobrevivência de C. aquaticum (imaturos/ninfas) por dia de experimentação e aceitação em teste de aceitabilidade alimentar com 12 espécies de macrófitas aquáticas. Legendas: $\mathrm{N}=$ Número de imaturos/ninfas testados em experimento com uma repetição; $\mathrm{X}=$ média de sobrevivência por dia; Dp = desvio padrão.

\begin{tabular}{|c|c|c|c|c|c|c|c|c|c|c|c|c|c|c|c|c|c|c|c|c|c|c|c|c|}
\hline \multirow{3}{*}{$\begin{array}{l}\text { Macrófitas } \\
\text { aquáticas }\end{array}$} & \multirow{3}{*}{$\mathrm{N}$} & \multicolumn{23}{|c|}{ Número de dias de experimento } \\
\hline & & \multicolumn{2}{|c|}{$2^{\circ}$} & \multicolumn{2}{|c|}{$4^{\circ}$} & \multicolumn{2}{|c|}{$6^{\circ}$} & \multicolumn{2}{|c|}{$8^{\circ}$} & \multicolumn{2}{|c|}{$10^{\circ}$} & \multicolumn{2}{|c|}{$12^{\circ}$} & \multicolumn{2}{|c|}{$14^{\circ}$} & \multicolumn{2}{|c|}{$16^{\circ}$} & \multicolumn{2}{|c|}{$18^{\circ}$} & \multicolumn{2}{|c|}{$20^{\circ}$} & \multicolumn{2}{|c|}{$21^{\circ}$} & \multirow{2}{*}{ 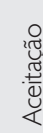 } \\
\hline & & $\times$ & ค̊ & $\times$ & ค̊ & $\times$ & $\stackrel{\circ}{0}$ & $\times$ & $\stackrel{\circ}{0}$ & $\times$ & $\stackrel{\circ}{0}$ & $\times$ & คి & $\times$ & $\stackrel{\circ}{0}$ & $x$ & $\stackrel{\circ}{0}$ & $\times$ & ค̊ & $\times$ & ๑̊ & $\times$ & $\stackrel{\circ}{0}$ & \\
\hline $\begin{array}{c}\text { Eichhornia } \\
\text { crassipes }\end{array}$ & 20 & 10 & 0 & 10 & 0 & 10 & 0 & 9 & 0 & 8.5 & 0.7 & 8 & 1.7 & 7 & 0 & 7 & 0 & 7 & 0 & 7 & 0 & 7 & 0 & + \\
\hline Paspalum sp. & 20 & 10 & 0 & 8.5 & 0.7 & 7 & 0 & 5 & 0 & 2 & 1.4 & 1 & 0 & 0 & 0 & 0 & 0 & 0 & 0 & 0 & 0 & 0 & 0 & - \\
\hline Pistia stratiotes & 20 & 10 & 0 & 7 & 0 & 5.5 & 0.7 & 4.5 & 0.7 & 4 & 1.4 & 4 & 1.4 & 3.5 & 0.7 & 3 & 0 & 2.5 & 0.7 & 2 & 0 & 2 & 0 & + \\
\hline $\begin{array}{l}\text { Polygonum } \\
\text { acuminatum }\end{array}$ & 20 & 10 & 0 & 5.5 & 0.7 & 2.5 & 0.7 & 0 & 0 & 0 & 0 & 0 & 0 & 0 & 0 & 0 & 0 & 0 & 0 & 0 & 0 & 0 & 0 & - \\
\hline $\begin{array}{l}\text { Hymenachne } \\
\text { amplexicaulis }\end{array}$ & 20 & 10 & 0 & 0 & 0 & 0 & 0 & 0 & 0 & 0 & 0 & 0 & 0 & 0 & 0 & 0 & 0 & 0 & 0 & 0 & 0 & 0 & 0 & - \\
\hline $\begin{array}{c}\text { Ludwigia } \\
\text { helminthorrhiza }\end{array}$ & 20 & 10 & 0 & 2.5 & 0.7 & 1 & 1.4 & 0 & 0 & 0 & 0 & 0 & 0 & 0 & 0 & 0 & 0 & 0 & 0 & 0 & 0 & 0 & 0 & - \\
\hline $\begin{array}{l}\text { Nymphoides } \\
\text { indica }\end{array}$ & 20 & 10 & 0 & 2 & 0 & 0 & 0 & 0 & 0 & 0 & 0 & 0 & 0 & 0 & 0 & 0 & 0 & 0 & 0 & 0 & 0 & 0 & 0 & - \\
\hline $\begin{array}{c}\text { Nymphaea } \\
\text { ampla }\end{array}$ & 20 & 9.5 & 0.7 & 0 & 0 & 0 & 0 & 0 & 0 & 0 & 0 & 0 & 0 & 0 & 0 & 0 & 0 & 0 & 0 & 0 & 0 & 0 & 0 & - \\
\hline $\begin{array}{c}\text { Nothoscordum } \\
\text { sp. }\end{array}$ & 20 & 10 & 0 & 9.5 & 0.7 & 8.5 & 0.7 & 8 & 0 & 7.5 & 0.7 & 7 & 1.4 & 6.5 & 2.1 & 6.5 & 2.1 & 3.5 & 0.7 & 3.5 & 0.7 & 3.5 & 0.7 & + \\
\hline $\begin{array}{l}\text { Eleocharis } \\
\text { acutangula }\end{array}$ & 20 & 10 & 0 & 6.5 & 0.7 & 2 & 1.4 & 0.5 & 0.7 & 0 & 0 & 0 & 0 & 0 & 0 & 0 & 0 & 0 & 0 & 0 & 0 & 0 & 0 & - \\
\hline $\begin{array}{c}\text { Salvinia } \\
\text { auriculata }\end{array}$ & 20 & 10 & 0 & 5.5 & 0.7 & 1 & 0 & 1 & 0 & 0.5 & 0.7 & 0 & 0 & 0 & 0 & 0 & 0 & 0 & 0 & 0 & 0 & 0 & 0 & - \\
\hline $\begin{array}{c}\text { Polygonum } \\
\text { hydropiperoides }\end{array}$ & 20 & 7.5 & 0.7 & 2.5 & 2.1 & 1.5 & 0.7 & 0.5 & 0.7 & 0 & 0 & 0 & 0 & 0 & 0 & 0 & 0 & 0 & 0 & 0 & 0 & 0 & 0 & - \\
\hline
\end{tabular}



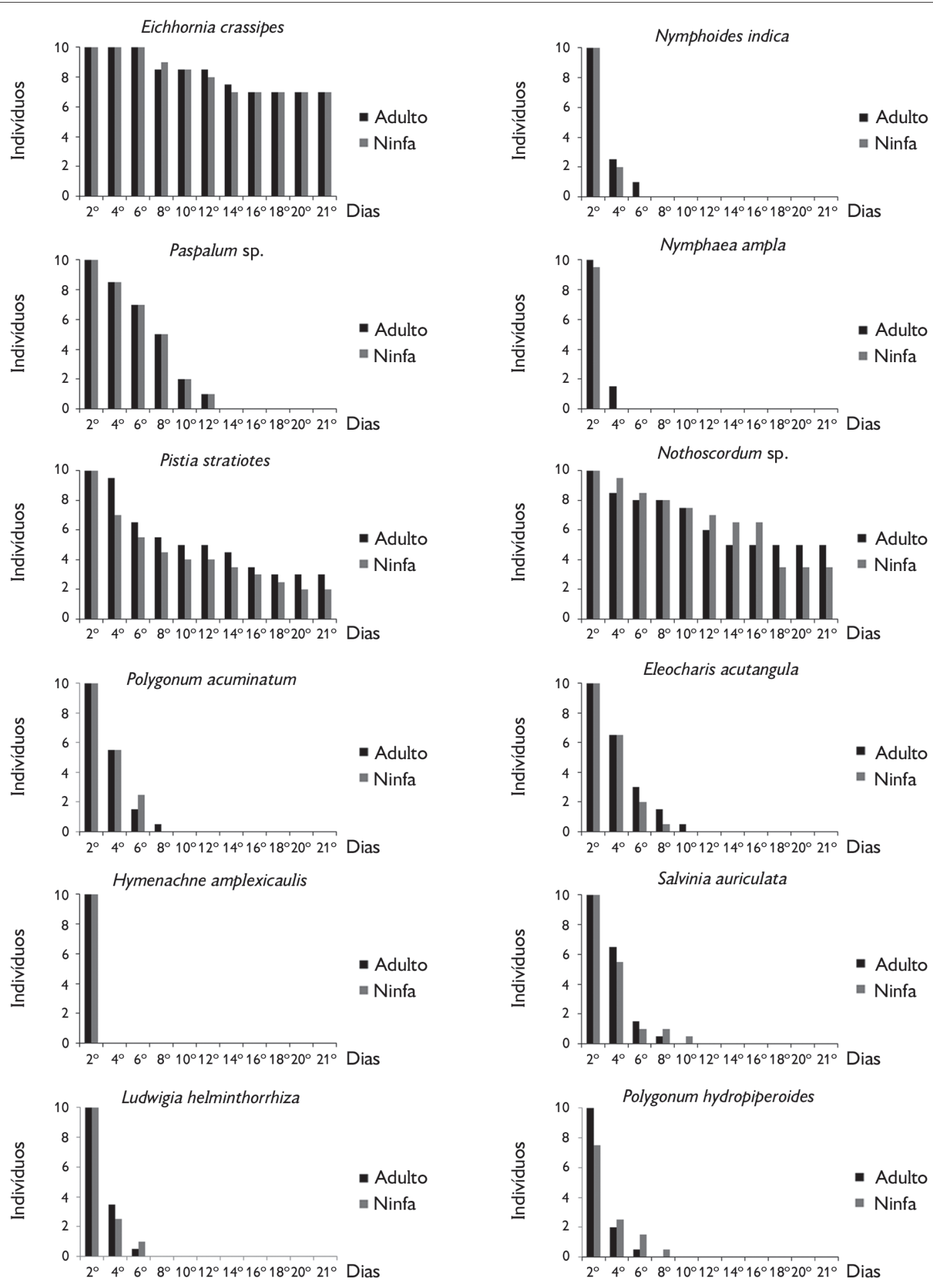

Figura 2. Sobrevivência de adultos e de imaturos (ninfas) de C. aquaticum em teste de aceitabilidade alimentar com 12 espécies de macrófitas aquáticas.

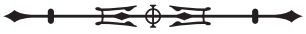


Tal condição foi observada para C. aquaticum na Argentina (Capello et al., 2012), cujos consumo e variação alimentar desse gafanhoto são diretamente influenciados pelas estações do ano naqueles ambientes (Franceschini et al., 2013), e também para outras espécies de gafanhotos semiaquáticos (Paulinia acuminata e Stenacris fissicauda fissicauda), que se alimentaram de macrófitas aquáticas não hospedeiras de forma temporária (alimentação alternativa), em estudos realizados na Amazônia central (Nunes, 1989; Vieira \& Adis, 2002), onde o pulso de inundação rege as dinâmicas hídricas e biológicas dessa região (Junk et al., 1989).

Quanto ao exposto anteriormente, em relação às espécies Eleocharis acutangula, Nymphoides indica e Salvinia auriculata e o gênero Ludwigia, que não foram aceitas pelo gafanhoto neste trabalho, ressalta-se que, em estudo realizado por Ferreira \& Vasconcellos-Neto (2001) no Pantanal do Mato Grosso, por meio da análise de conteúdo estomacal, os fragmentos de tais plantas foram encontrados no trato digestivo de espécimes de $C$. aquaticum oriundos de campo. Isso indica que houve consumo dessas plantas pelo gafanhoto quando em ambiente natural. Em insetos fitófagos, a preferência alimentar parece não estar relacionada apenas ao fato de eles mastigarem ou consumirem esporadicamente um determinado vegetal, mas sim na dependência da estrutura da planta (pilosidade, dureza da folha), presença de substâncias químicas, como os fago-estimulantes, e de substâncias impeditivas (Nunes, 1989) ou tóxicas (Vendramim \& Guzzo, 2009).

Além disso, Capello et al. (2012), em estudo na Argentina, indicam que $C$. aquaticum possui uma tendência à polifagia, com preferência alimentar por E. crassipes, mas que pode se alimentar esporadicamente de outras macrófitas. Também deve-se considerar que o consumo e a variabilidade alimentar de $C$. aquaticum podem variar quanto aos estágios (adulto e ninfa) e ao sexo, visto que as fêmeas tendem a apresentar uma maior amplitude alimentar do que os machos e os imaturos, assim como as ninfas em instares iniciais (do $1^{\circ}$ ao $3^{\circ}$ instares) tendem a consumir somente a planta hospedeira principal, e nos demais $\left(4^{\circ}, 5^{\circ}\right.$ e $6^{\circ}$ instares) esporadicamente outras macrófitas (Capello et al., 2011).

No entanto, deve-se destacar que tais constatações foram feitas em outros ambientes, sabendo-se que o consumo e a variabilidade alimentar de uma mesma espécie podem sofrer mudanças ao longo do ano e também em regiões distintas (Franceschini et al., 2013). Desse modo, os padrões observados em determinadas localidades podem não ser os mesmos de outras, como as observadas neste estudo, no Parque Ecológico do Utinga (PEUt), na Amazônia oriental.

Com relação à presença de substâncias tóxicas existentes nas plantas, ressalta-se que a não aceitação de Polygonum hydropiperoides (pimenta-do-brejo) por C. aquaticum possivelmente foi decorrente de esta macrófita apresentar um suco fortemente acre (ácido) e picante, sendo por alguns estudiosos considerado tóxico (Lorenzi, 2008), o que explicaria a morte rápida dos gafanhotos no teste de aceitabilidade realizado.

O maior índice de sobrevivência de C. aquaticum durante o experimento foi observado para os gafanhotos (adultos e ninfas) que se alimentaram de E. crassipes, com $70 \%$ de sobrevivência, tanto para adultos quanto para ninfas.

Considerando que C. aquaticum apresenta especificidade alimentar e hospedeira nesta planta (Ferreira \& Vasconcellos-Neto, 2001; Braga et al., 2013), o resultado obtido neste estudo quanto às mortes dos gafanhotos testados em E. crassipes apontou para a possibilidade de se considerar que elas provavelmente foram decorrentes da idade (longevidade) dos adultos testados e da deficiência no processo de muda dos imaturos, visto que na troca da cutícula, muitas vezes, o aparelho bucal das ninfas ficam presos no exoesqueleto velho, impedindo-as de se alimentarem (Nunes, 1989).

Ressalte-se que apenas para as macrófitas E. crassipes, Nothoscordum sp. e Pistia stratiotes registraram-se gafanhotos vivos e se alimentando destas durante os 21 dias de experimentação, nas demais plantas (outras noves macrófitas) não ocorreram indivíduos vivos ao término 
dos testes, sendo portanto consideradas não aceitas como alimento pelo gafanhoto. Até a $7^{\mathrm{a}}$ observação ( $14^{\circ}$ dia de experimento), $75 \%$ das plantas testadas não apresentavam mais indivíduos vivos (Tabela 3, Figura 2).

Entre as espécies de macrófitas não aceitas, a maior mortandade, em curto espaço de tempo (2a observação/ quatro dias de teste/19\% do tempo de teste), foi observada nas plantas Hymenachne amplexicaulis, com 100\% de mortalidade para ninfas e adultos, Nymphaea ampla, com 92,5\% de mortalidade (100\% para ninfas e $85 \%$ para adultos), e Nymphoides indica, com $77,5 \%$ de mortalidade (80\% para ninfas e $75 \%$ para adultos), o que parece indicar a existência de alguma substância que causa a maior nocividade destas plantas para C. aquaticum. Entretanto, somente uma análise química específica seria capaz de acusar a presença de substâncias que poderiam, por exemplo, ser tóxicas ao gafanhoto. A espécie Paspalum sp. mostrou-se menos nociva ao gafanhoto, visto que até a $6^{a}$ observação ( 12 dias de teste/57\% do tempo de experimento) ainda havia espécimes vivos (Tabelas 1 e 2; Figura 2). Essas observações durante o desenvolvimento do experimento comprovam que os gafanhotos adultos e imaturos comeram essas macrófitas e até mesmo defecaram o alimento ingerido. Quanto a isso, vale ressaltar que a rápida mortandade causada a insetos herbívoros por algumas plantas é ocasionada por uma concentração de sílica e lignina na epiderme das plantas (Vendramim \& Guzzo, 2009).

A sílica é um elemento que causa dureza às folhas das plantas, o que prejudica a mastigação, a ingestão, a digestibilidade e impede a conversão do alimento ingerido, causando um desbalanceamento nutricional,

Tabela 3. Sobrevivência absoluta, percentual e total de adultos e de ninfas de C. aquaticum em dois experimentos de aceitabilidade alimentar em 12 espécies de macrófitas aquáticas.

\begin{tabular}{|c|c|c|c|c|c|c|c|c|}
\hline \multirow[b]{2}{*}{ Macrófitas aquáticas } & \multicolumn{3}{|c|}{ Adultos } & \multicolumn{3}{|c|}{ Ninfas } & \multirow[b]{2}{*}{$\begin{array}{c}\text { Total de } \\
\text { indivíduos } \\
\text { vivos }\end{array}$} & \multirow[b]{2}{*}{$\begin{array}{l}\text { Índice de } \\
\text { sobrevivência } \\
\text { geral }\end{array}$} \\
\hline & $\begin{array}{l}\text { Número de } \\
\text { indivíduos } \\
\text { testados }\end{array}$ & $\begin{array}{l}\text { Número de } \\
\text { indivíduos } \\
\text { vivos }\end{array}$ & $\begin{array}{c}\text { Índice de } \\
\text { sobrevivência }\end{array}$ & $\begin{array}{c}\text { Número de } \\
\text { indivíduos } \\
\text { testados }\end{array}$ & $\begin{array}{l}\text { Número de } \\
\text { indivíduos } \\
\text { vivos }\end{array}$ & $\begin{array}{c}\text { Índice de } \\
\text { sobrevivência }\end{array}$ & & \\
\hline Eichhornia crassipes & 20 & 14 & $70 \%$ & 20 & 14 & $70 \%$ & 28 & $70 \%$ \\
\hline Paspalum sp. & 20 & 0 & $0 \%$ & 20 & 0 & $0 \%$ & 0 & $0 \%$ \\
\hline Pistia stratiotes & 20 & 6 & $30 \%$ & 20 & 4 & $20 \%$ & 10 & $25 \%$ \\
\hline $\begin{array}{l}\text { Polygonum } \\
\text { acuminatum }\end{array}$ & 20 & 0 & $0 \%$ & 20 & 0 & $0 \%$ & 0 & $0 \%$ \\
\hline $\begin{array}{l}\text { Hymenachne } \\
\text { amplexicaulis }\end{array}$ & 20 & 0 & $0 \%$ & 20 & 0 & $0 \%$ & 0 & $0 \%$ \\
\hline $\begin{array}{c}\text { Ludwigia } \\
\text { helminthorrhiza }\end{array}$ & 20 & 0 & $0 \%$ & 20 & 0 & $0 \%$ & 0 & $0 \%$ \\
\hline Nymphoides indica & 20 & 0 & $0 \%$ & 20 & 0 & $0 \%$ & 0 & $0 \%$ \\
\hline Nymphaea ampla & 20 & 0 & $0 \%$ & 20 & 0 & $0 \%$ & 0 & $0 \%$ \\
\hline Nothoscordum sp. & 20 & 10 & $50 \%$ & 20 & 7 & $35 \%$ & 17 & $42.5 \%$ \\
\hline Eleocharis acutangula & 20 & 0 & $0 \%$ & 20 & 0 & $0 \%$ & 0 & $0 \%$ \\
\hline Salvinia auriculata & 20 & 0 & $0 \%$ & 20 & 0 & $0 \%$ & 0 & $0 \%$ \\
\hline $\begin{array}{c}\text { Polygonum } \\
\text { hydropiperoides }\end{array}$ & 20 & 0 & $0 \%$ & 20 & 0 & $0 \%$ & 0 & $0 \%$ \\
\hline
\end{tabular}


enquanto a lignina é uma substância que, em determinadas concentrações nas plantas, é extremamente tóxica para os insetos, causando a morte em poucos dias (Vendramim \& Guzzo, 2009). Pelo exposto, pode-se admitir que as plantas mencionadas, que apresentaram maior mortandade no início do experimento, podem conter em suas epidermes os elementos sílica e lignina, entretanto apenas uma análise química específica das folhas dessas plantas poderia comprovar tal suposição.

No experimento, foi registrada a ocorrência de processos de muda sofrida pelas ninfas de $C$. aquaticum em todas as espécies de macrófitas testadas. Esse processo é responsável pelo crescimento dos insetos e corresponde à troca total do exoesqueleto destes animais. É um processo que requer grande gasto energético (Borror \& Delong, 1988; Triplehorn \& Jonnson, 2011). As ecdises (mudas) observadas aconteceram em dias diferentes nos testes, sendo a maioria registrada nos dias iniciais do experimento (até o $4^{\circ} \mathrm{dia}$ ), o que possivelmente é decorrente de reservas energéticas adquiridas pelas ninfas antes do experimento, quando elas ainda estavam em campo. Entretanto, apenas com a macrófita $E$. crassipes ocorreram mudas a partir do $4^{\circ}$ até o $21^{\circ}$ dia de teste, o que torna possível admitir que tais processos ocorreram com as reservas energéticas provenientes dessa planta aquática oferecida como alimentação aos gafanhotos, e isso reforça a aceitabilidade dessa macrófita por $C$. aquaticum.

Neste estudo, adultos e ninfas de C. aquaticum alimentaram-se de todas as macrófitas aquáticas utilizadas, mesmo que em algumas plantas, em poucos dias, os gafanhotos tenham morrido. Quanto a isso, Bernays \& Bright (1991) afirmam que a necessidade de herbívoros se alimentarem de outras plantas não preferenciais pode estar relacionada com o equilíbrio da quantidade de água que deve ser mantida no corpo dos insetos, e não a uma necessidade ou preferência alimentar. Entretanto, essa afirmação não se aplica aos experimentos realizados, pois os béqueres de vidro, onde foram acondicionados os gafanhotos durante o teste, encontravam-se forrados com algodão embebido em água, propiciando sempre uma linha de água suficiente para garantir a maior umidade possível a eles, visto que se tratavam de insetos semiaquáticos com grande dependência do meio aquático.

A não aceitação das nove espécies macrófitas aquáticas testadas parece ser decorrente de fatores relacionados à resistência das plantas aos ataques de insetos e, nesse caso, o tipo mais adequado para justificar tal resultado, segundo a classificação de Vendramim \& Guzzo (2009), é a antibiose, visto que os gafanhotos que se alimentaram dessas plantas não sobreviveram. Entre as três plantas aceitas nos testes, E. crassipes foi considerada a macrófita preferida pelos gafanhotos, tendo em vista que nela houve a maior sobrevivência desses insetos, devido à relação hospedeira de $C$. aquaticum com esta espécie vegetal, o que é corroborado pelos estudos de Adis et al. (2007), Braga et al. (2011) e Capello et al. (2012).

Alguns estudos indicam que a relação hospedeira existente entre plantas e insetos pode ser explicada por processos coevolutivos entre esses organismos, onde ambos desenvolvem diferentes estratégias para manter a relação inseto-planta existente hoje em dia (Ehrlich \& Raven, 1964). Entretanto, para Lhano et al. (2005) e Braga et al. (2013), a aceitabilidade e a consequente relação hospedeira de C. aquaticum por E. crassipes devem-se ao fato de que esta macrófita reúne condições adequadas de alimento, proteção, abrigo, substrato para as oviposições endofíticas e também para o desenvolvimento do ciclo de vida desse gafanhoto, fato que não ocorre com as demais macrófitas aquáticas, apesar de algumas serem utilizadas esporadicamente como fonte alternativa para sua alimentação.

Quanto à possibilidade do uso de C. aquaticum como controlador de macrófitas aquáticas nos lagos Bolonha e Água Preta, o presente estudo indica que, além de Eichhornia crassipes ser a planta preferida do gafanhoto, existe a possibilidade de também ser usada para controlar a presença de Nothoscordum sp. e Pistia stratiotes nesses lagos, as quais tiveram significativa aceitação por $C$. 
aquaticum, o que não quer dizer que em outros ambientes ou regiões Nothoscordum sp. e P. stratiotes possam ser controladas por C. aquaticum. Para que tal utilização seja concretizada, no entanto, é necessário que estudos ou experimentos específicos para esse fim sejam realizados previamente nos lagos Bolonha e Água Preta, levando-se em consideração o consumo alimentar do gafanhoto $C$. aquaticum, para que seja determinada a quantidade de indivíduos (gafanhotos) que devem ser usados no controle destas macrófitas, tal como o realizado em lagos naturais na Amazônia central por Braga et al. (2013). Neste estudo, o autor identificou que a proporção de 22 a 89 indivíduos por metro quadrado mostrou-se bastante eficiente no controle de E. crassipes em experimento monitorado. É sabido que, apesar de C. aquaticum ser capaz de causar grandes danos à sua macrófita hospedeira, esta espécie não é suficiente para impedir o crescimento de $E$. crassipes, devido à alta taxa de crescimento desta planta e à baixa abundância de C. aquaticum no ambiente natural deste vegetal (Franceschini et al., 2011).

\section{CONCLUSÃO}

Este estudo evidenciou a aceitação alimentar de Cornops aquaticum pelas macrófitas aquáticas Eichhornia crassipes, Nothoscordum sp. e Pistia stratiotes entre 12 espécies testadas. Esta informação é importante e imprescindível para a utilização desse gafanhoto como um possível agente de controle biológico dessas macrófitas aquáticas, especialmente E. crassipes, nos lagos Bolonha e Água Preta, no Parque Ecológico do Utinga (PEUt). Entretanto, para a utilização de $C$. aquaticum no controle dessas macrófitas, são ainda necessários estudos complementares, referentes à quantificação de gafanhotos no consumo dessas plantas, para comprovar o potencial desse inseto no controle dessas macrófitas aquáticas no PEUt.

A maior sobrevivência de adultos e imaturos (ninfas) de C. aquaticum, entre as três plantas aceitas, foi observada para E. crassipes, enquanto a maior mortalidade dos gafanhotos ocorreu nas macrófitas Hymenachne amplexicaulis, Nymphaea ampla e Nymphoides indica, sugerindo que tais plantas parecem ser mais nocivas entres as que efetivamente o gafanhoto comeu durante os testes. Também foi feito o primeiro registro de aceitação alimentar de $C$. aquaticum por uma espécie de macrófita aquática do gênero Nothoscordum.

\section{REFERÊNCIAS}

ADIS, J. \& W. J. JUNK, 2003. Feeding impact and bionomics of the grasshopper Cornops aquaticum on the water hyacinth Eichhornia crassipes in Central Amazonian Floodplains. Studies on Neotropical Fauna and Environment 38(3): 245-249.

ADIS, J., E. BUSTORF, M. G. LHANO, C. AMEDEGNATO \& A. L. NUNES, 2007. Distribution of Cornops grasshoppers (Leptysminae: Acrididae: Orthoptera) in Latin America and the Caribbean Islands. Studies on Neotropical Fauna and Environment 42(1): 11-24.

BENTOS-PEREIRA, A. \& E. LORIER, 1991. Acridomorfos acuaticos (Orthoptera, Acridoidea). 1. Adaptaciones morfologicas. Revista Brasileira de Entomologia 35(3): 631-653.

BERNAYS, E. A. \& K. L. BRIGHT, 1991. Dietary mixing in grasshoppers: switching induced by nutritional imbalances in foods. Entomologia Experimentalis et Applicata 61(3): 247-253.

BORROR, D. J. \& D. M. DELONG, 1988. Introdução ao estudo dos insetos: 1-653. Edgard Blucher, São Paulo.

BRAGA, C. E., A. L. NUNES-GUTJAHR, J. W. MORAIS \& J. ADIS, 2011. Fenologia de Cornops aquaticum (Bruner 1906) (Orthoptera: Acrididae) associado a Eichhornia crassipes (Pontederiaceae) em um Lago de Várzea na Amazônia Central, Brasil. Revista de la Sociedad Entomológica Argentina 70(3-4): 185-196.

BRAGA, C. E., A. L. NUNES, J. W. MORAIS \& J. ADIS, 2013. Avaliação do potencial do gafanhoto Cornops aquaticum (Orthoptera) como agente de controle biológico de Eichhornia crassipes (Pontederiaceae). Interciencia 38(8): 590-596.

BRITO, S. K. M., 2009. Parque Estadual do Utinga (PA): uma gestão ambiental participativa: 1-102. Dissertação (Mestrado em Gestão de Recursos Naturais e Desenvolvimento Local na Amazônia) - Universidade Federal do Pará, Belém.

CAPELLO, S., M. L. DE WYSIECKI \& M. MARCHESE, 2011. Feeding patterns of the aquatic grasshopper Cornops aquaticum (Bruner) (Orthoptera: Acrididae) in the middle Paraná River, Argentina. Neotropical Entomology 40(2): 170-175.

CAPELLO, S., M. MARCHESE \& M. L. DE WYSIECKI, 2012. Feeding habits and trophic niche overlap of aquatic Orthoptera associated with macrophytes. Zoological Studies 51(1): 51-58. 
EHRLICH, P. R. \& P. H. RAVEN, 1964. Butterflies and plants: a study in coevolution. Evolution 18(4): 586-608.

FERREIRA, S. A. \& J. VASCONCELLOS-NETO, 2001. Plantas hospedeiras de Cornops aquaticum (Bruner) (Orthoptera: Acrididae) no Pantanal de Poconé-MT. Neotropical Entomology 30(4): 523-533.

FRANCESCHINI, C., M. L. DE WYSIECKI, A. POI DE NEFF, M. E. GALASSI \& F. S. MARTINEZ, 2011. The role of the host-specific grasshopper Cornops aquaticum (Orthoptera: Acrididae) as consumer of native Eichhornia crassipes (Pontederiaceae) floating meadows. Revista de Biología Tropical 59(3): 1407-1418.

FRANCESCHINI, M. C., M. L. DE WYSIECKI \& A. POI, 2013. Age structure and feeding of the neotropical grasshopper Cornops aquaticum (Bruner) (Orthoptera: Acrididae) on water hyacinth. Neotropical Entomology 42(4): 344-350.

GUIDO, A. S. \& B. D. PERKINS, 1975. Biology and host specificity of Cornops aquaticum (BRUNER) (Orthoptera: Acrididae), a potential biological control agent of water hyacinth. Environmental Entomology 4(3): 400-404.

HILL, M. P. \& C. J. CILLIERS, 1999. A review of the arthropod natural enemies, and factors that influence their efficacy, in the biological control of water hycinth, Eichhornia crassipes (Mart.) SolmsLauterbach (Pontederiacae), in South Africa. African Entomology Memoirs 1: 89-102.

HILL, M. P. \& I. G. OBERHOLZER, 2000. Host specificity of the grassholpper Cornops aquaticum, a natural enemy of water hyacinth. Proceedings of the International Symposium on Biological Control of Weeds 10: 349-356.

JUNK, W. J., P. B. BAYLEY \& R. E. SPARKS, 1989. The flood pulse concept in river-floodplains systems. Canadian Journal of Fishers and Aquatic 106: 110-127.

LHANO, M. G., J. ADIS, M. I. MARQUES \& L. D. BATTIROLA, 2005. Cornops aquaticum (Orthoptera, Acrididae, Leptysminae): aceitação de plantas alimentares por ninfas vivendo em Eichhornia azurea (Pontederiaceae) no Pantanal Norte, Brasil. Amazoniana 18(3-4): 397-404.

LORENZI, H., 2008. Plantas daninhas do Brasil: terrestres, aquáticas, parasitas e tóxicas. Instituto Plantarum de Estudos da Flora, Nova Odessa.
NUNES, A. L., 1989. Estudo sobre o ciclo de vida e fenologia de Stenacris fissicauda fissicauda (Bruner, 1908) (OrthopteraAcrididae) em um lago de várzea da Amazônia Central, Brasil, Manaus: 1-122. Dissertação (Mestrado em Ciências Biológicas: Entomologia) - Instituto Nacional de Pesquisas da Amazônia/ Universidade Federal do Amazonas, Manaus.

NUNES-GUTJAHR, A. L. \& C. E. BRAGA, 2011. Gafanhotos. In: M. L. OLIVEIRA, F. B. BACCARO, R. BRAGA-NETO \&, W. E. MAGNUSSON (Orgs.): Reserva Ducke: a biodiversidade amazônica através de uma grade: 131-143. Áttema Design Editorial, Manaus.

ROBERTS, H. R. \& C. S. CARBONELL, 1979. A revision of the genera Stenopola and Cornops (Orthoptera, Acrididae, Leptysminae). Proceeding of the Academy of Natural Sciences of Philadelphia 131: 104-130.

SILVA, F. R. J., M. I. MARQUES, L. D. BATTIROLA \& M. G. LHANO, 2010. Fenologia de Cornops aquaticum (Bruner) (Orthoptera: Acrididae) em Eichhornia azurea (Pontederiaceae) no norte do Pantanal de Mato Grosso. Neotropical Entomology 39(4): 535-542.

TRIPLEHORN, C. A. \& N. F. JONNSON, 2011. Estudos dos insetos: tradução da $7^{\text {a }}$ edição de Borror and Delong's introduction to the study of insects. Cengage Learning, São Paulo.

VENDRAMIM, J. D. \& E. C. GUZZO, 2009. Resistência de plantas e a bioecologia e nutrição dos insetos. In: A. R. PANIZZI \& J. R. P. PARRA (Eds.): Bioecologia e nutrição dos insetos: base para o manejo integrado de pragas: 1055-1105. Embrapa Informação Tecnológica, Brasília.

VIEIRA, M. F. \& J. ADIS, 2002. Aceitabilidade alimentar de Paulinia acuminata (De Geer, 1773) (Orthoptera: Pauliniidae) na várzea da Amazônia Central. Acta Amazonica 32(2): 333-338.

VIEIRA, M. F. \& A. C. SANTOS, 2003. Duração do ciclo de vida de Cornops aquaticum (Bruner, 1906) (Orthoptera: Acrididae: Leptysminae) e aspectos de seu comportamento alimentar na Amazônia central. Acta Amazonica 33(4): 711-714.

ZOLESSI, L. C., 1956. Observaciones sobre Cornops aquaticum Br. (Acridoidea, Catantopinae) en el Uruguay. Revista de la Sociedad Uruguaya de Entomologia 1: 3-28.

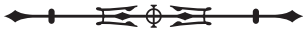


